\title{
Microfluidic cell sorter with integrated piezoelectric actuator
}

\author{
Chun H. Chen • Sung Hwan Cho • Frank Tsai • \\ Ahmet Erten - Yu-Hwa Lo
}

Published online: 1 August 2009

(C) The Author(s) 2009. This article is published with open access at Springerlink.com

\begin{abstract}
We demonstrate a low-power $(<0.1 \mathrm{~mW})$, low-voltage $\left(<10 \mathrm{~V}_{\mathrm{p}-\mathrm{p}}\right)$ on-chip piezoelectrically actuated micro-sorter that can deflect single particles and cells at high-speed. With rhodamine in the stream, switching of flow between channels can be visualized at high actuation frequency $(\sim 1.7 \mathrm{kHz})$. The magnitude of the cell deflection can be precisely controlled by the magnitude and waveform of input voltage. Both simulation and experimental results indicate that the drag force imposed on the suspended particle/cell by the instantaneous fluid displacement can alter the trajectory of the particle/cell of any size, shape, and density of interest in a controlled manner. The openloop E. Coli cell deflection experiment demonstrates that the sorting mechanism can produce a throughput of at least 330 cells/s, with a promise of a significantly higher throughput for an optimized design. To achieve close-loop sorting operation, fluorescence detection, real-time signal processing, and field-programmable-gate-array (FPGA) implementation of the control algorithms were developed to perform automated sorting of fluorescent beads. The preliminary results show error-free sorting at a sorting
\end{abstract}

\section{H. Chen $(\square)$}

Department of Bioengineering,

University of California at San Diego,

La Jolla, CA 92093, USA

e-mail: chc008@gmail.com

S. Cho

Department of Material Science,

University of California at San Diego,

La Jolla, CA 92093, USA

F. Tsai $\cdot$ A. Erten $\cdot$ Y.-H. Lo

Department of Electrical Engineering,

University of California at San Diego,

La Jolla, CA 92093, USA efficiency of $\sim 70 \%$. Since the piezoelectric actuator has an intrinsic response time of $0.1-1 \mathrm{~ms}$ and the sorting can be performed under high flowrate (particle speed of $\sim 1-10 \mathrm{~cm} / \mathrm{s}$ ), the system can achieve a throughput of $>1,000$ particles/s with high purity.

Keywords Hydrodynamics · Flow cytometer .

Microfluidics $\cdot$ Sorter $\cdot$ Piezoelectric actuation

\section{Introduction}

Compared to conventional fluorescence-activated cell sorter (FACS), $\mu$ FACS has the advantages of lower cost, reduced reagent usage, portability and rapid analysis time, which make them attractive for various biotechnological applications (e.g. bioanalysis and drug discovery) (Dittrich 2003). Due to the growing interest in the study of single living cells (e.g. effect of drugs and gene expression studies in cancer research (Sims and Allbritton 2007; Andersson and Berg 2003; Meredith et al. 2000), along with advances in microfabrication technology, significant progress has been made toward developing $\mu$ FACS that can sort single cells with high efficiency and throughput.

Numerous types of $\mu$ FACS based on the principle of continuous flow separation (i.e. continuous injection, monitoring detection, and sorting based on spatial separation (Pamme 2007) have been developed to sort various types of cells including yeast, bacterial, and blood cells. Among those published micro sorting techniques are electroosmotic (Fu et al. 2004; Fu et al. 1999), dielectrophoretic (DEP) (Lapizco-Encinas et al. 2004; Fiedler et al. 1998; Holmes et al. 2005; Doh and Cho 2005; Braschler et al. 2007; Hu et al. 2005), magnetic (Pamme 2006; Pamme and Wilhelm 2006), and hydrodynamic (Bang et al. 2006; 
Krüger et al. 2002; Wolff et al. 2003) sorting. Electroosmotic sorters can achieve precise flow switching, but such devices operate under high DC voltage (e.g. hundreds of volts), require frequent change of voltage settings due to ion depletion ( $\mathrm{Fu}$ 2002), and suffer from low sorting rate (e.g. tens of particles per sec). On the other hand, DEPbased sorting exhibits precise manipulation down to single cell level, but the fabrication is highly complex and the cell differentiation capability is rather limited. For DEP-based sorters, particles and cells with similar dielectric properties cannot be easily separated from one another since they experience similar DEP forces. Magnetic sorting demonstrates high selectivity but requires labeling of magnetic particles, which cause irreversible cell damages. The aforementioned drawbacks may be resolved with hydrodynamic flow-switching methods such as sheath flow steering (Blankenstein and Lasen 1998) and integration of membrane valve to alter fluid flow (Fu et al. 2002). However, these techniques have long response time due to the inherent properties of the mechanical components. As a result, the sorting rate of these devices is low in general. Due to the advance in check valve technology, several groups (Bang et al. 2006, Wolff et al. 2003) have implemented high-speed off-chip flow-switching check valve (i.e. response time of $2.5 \mathrm{~ms}$ ) to enhance sorting throughput $(\sim 12,000$ cells/sec). However, due to the lack of precise electronics control and large electronics-induced timing jitter, both the recovery rate and purity of sorting can be low. Also, the use of bulky external actuators (syringe pumps, external check valves, etc) in this sorting scheme hinders miniaturization.

In this paper, we describe a micro-sorter with a piezoelectric/metal bi-morphous actuator. The piezoelectric film is made of lead zirconate titanate (PZT), and the metal may be copper or stainless steel. The piezoelectric actuator has a diameter of $20 \mathrm{~mm}$ and an intrinsic resonant frequency of around $5 \mathrm{kHz}$. This PZT actuator is integrated with the microfluidic channels using UV ozone assisted bonding process to be discussed later. Compared to hybrid systems, the integrated approach offers several advantages: 1) low voltage and low power consumption $\left(<10 \mathrm{~V}_{\mathrm{p}-\mathrm{p}}\right.$ and $<0.1 \mathrm{~mW}$ ), 2) precise control of the magnitude of transverse cell/particle deflection to enable single particle/cell sorting, $3)$ intrinsically much faster response $(\sim 0.1-1 \mathrm{~ms})$ than conventional check or membrane valves. In addition, to suppress noise in fluorescence detection, we have designed a spatial filter and signal processing algorithm for signal-tonoise ratio enhancement and sorting verification (i.e. sorting error detection). To carry out automated sorting, characterize sorting efficiency and accuracy, and to reduce timing jitter to $<10 \mu \mathrm{sec}$, the signal processing algorithms as well as the actuator control algorithms have been implemented in field-programmable-gate-array (FPGA).

\section{Working principle}

\subsection{Sorting mechanism}

With a PZT actuator integrated on a chip, transverse displacement of fluid of the order of nanoliters can be introduced by the bending action (upward or downward) of the actuator controlled by the waveform of the input voltage (Fig. 1). As the targeted particle enters the sorting junction, the particle is being deflected transversely by the drag force of the displaced fluid and consequently follows the hydrodynamic fluid flow down to one of the collection channels. Without PZT actuation, unwanted particles travel straight down to the waste channel. To achieve the desirable amount of particle deflection, PZT actuator needs to provide a sufficient fluid volume displacement and a bending rate following the ramp of voltage. These needs are easily met in our system by adjusting input waveform and voltage magnitude for the PZT actuator.

\subsection{Dynamic simulation}

To visualize the trajectory of the particle subjecting to fluid deflection, a time-dependent simulation is conducted in

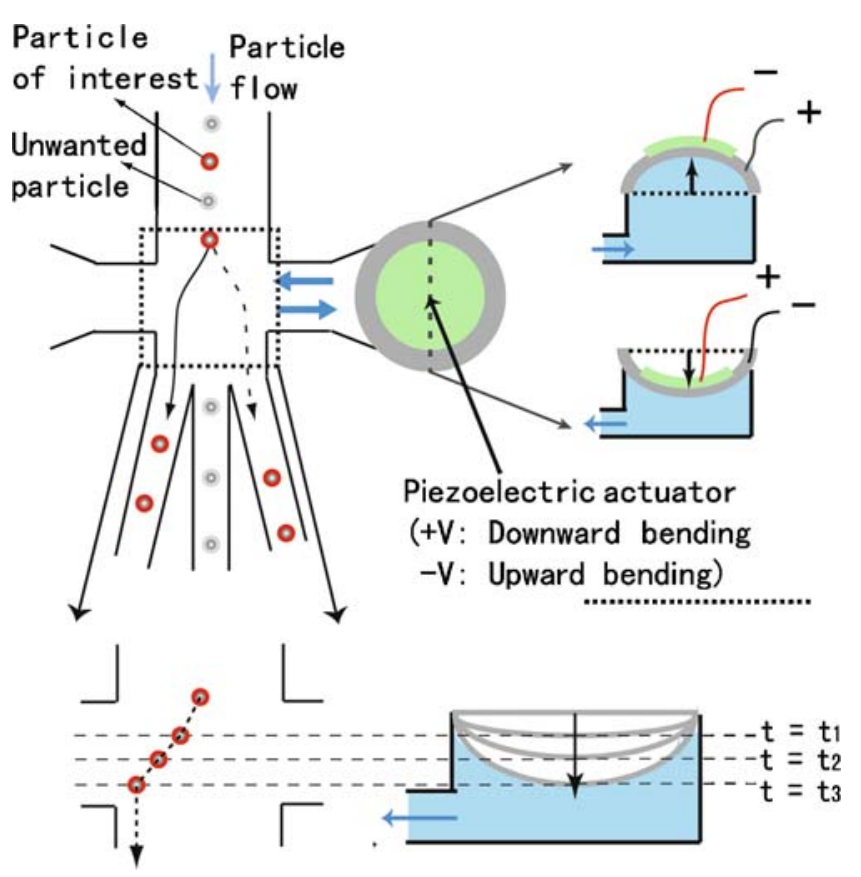

Fig. 1 Operating principle of the piezoelectric (PZT) sorter. As particle enters the sorting junction, bending motion of the PZT actuator will temporarily disturb fluid flow (either to the right or left), causing particles to be deflected to the left/right channels. The bending orientation (e.g. upward or downward) and the amount of bending of the PZT actuator are controlled by the polarity and the magnitude of the input voltage, respectively. In the absence of PZT actuation, unwanted particles stay in the center streamlines, which travel straight down to the waste channel 
Comsol 3.3 (Comsol Inc.). The dynamics of fluid flow is modeled using incompressible Navier-Stoke equation with water (with density $=1,000 \mathrm{~kg} / \mathrm{m}^{3}$ and viscosity $=1.002 \times$ $10^{-3}$ ) as the fluid. The inlet velocity profile is assumed to be parabolic with maximum center velocity of $5 \mathrm{~cm} / \mathrm{s}$ and the outlet channels assume atmosphere pressure. Transverse fluid deflection by the PZT actuation is modeled as timedependent sinusoidal pressure at the boundary wall of the sorting junction while rest of the channel walls assume rigid and no-slip boundary conditions. At the sorting junction, pressure is defined as

$\mathrm{P}_{\text {PZT_boundary }}=\mathrm{P}_{\mathrm{A}} \sin (2 \pi \mathrm{ft})+\mathrm{P}_{\mathrm{o}}$

$\mathrm{P}_{\mathrm{A}}$ is the actuation pressure, $\mathrm{t}$ is time, $\mathrm{f}$ is the actuation frequency, and $\mathrm{P}_{\mathrm{o}}$ is the hydrodynamic pressure established by the flow. To see the effect of PZT actuator-induced flow response, $5-\mu \mathrm{m}$ particles are released into the center of the flow stream at a velocity of $5 \mathrm{~cm} / \mathrm{s}$ and the movements of the particles are modeled using Khan and Richardson's force (Coulson et al. 1982). Time-dependent boundary parameters, $\mathrm{P}_{\mathrm{A}}, \mathrm{f}$, and $\mathrm{P}_{\mathrm{o}}$, are set at $1.5 \mathrm{kPa}, 250 \mathrm{~Hz}$, and $0.63 \mathrm{kPa}$ respectively.

\section{Experiment}

\subsection{Device fabrication}

The device is fabricated using the conventional micro replica molding technique (Xia and Whitesides 1998) in which device features are photolithographically defined by SU-8 photoresist mold (Fig. 2(a)) and then transferred to a polydimethylsiloxane (PDMS) substrate. For device assembly, the PDMS substrate and glass are surface treated in UV ozone chamber (Jelight Inc., Irvine) with a lamp output of $28 \mathrm{~mW}$ at $254 \mathrm{~nm}$. Bonding occurs as they are put into physical contact. For the subsequent PZT actuator and PDMS substrate bonding, the mechanically polished stainless steel surface of the PZT actuator is exposed to UV ozone treatment for $10 \mathrm{~min}$. After the actuation chamber of the PDMS substrate (Sylgard 184, Dow Corning) is created using a 16-mm diameter punch, both the PDMS substrate and the PZT actuator are UV ozone treated for another 5 min. Immediately after the UV o-zone treatment, the stainless steel surface of the PZT actuator is aligned to the PDMS actuation chamber and put in contact with the PDMS substrate for bonding. The bonding process is completed after the sample is baked at $85^{\circ} \mathrm{C}$ for $8 \mathrm{~h}$ (Fig. 2(b)). For the last step of device fabrication, Teflon tubings are inserted into the inlets and outlets of the device for fluid flow introduction. The device (Fig. 2(c)) is then primed using vacuuming filling under -30 inch $\mathrm{Hg}$. The strong stainless steel-to-PDMS bonding prevents fluid leakage and optimizes power coupling from the PZT actuator to the fluidic channels.

\subsection{Rhodamine and bead deflection}

The device is mounted on a microscope stage with a highspeed camera (Photron Fastcam, Photron Inc.) attached for visualization, and PZT actuation is driven by a function generator (Tektronix Inc.). $5 \mathrm{mM}$ of Rhodamine $6 \mathrm{G}$ is introduced to the sample channel and hydrodynamically focused (1:10 sample to sheath flow). Once the fluid flow is stabilized, the PZT actuator is electrically modulated and the behaviors of stream under various voltage and frequency are analyzed. Similarly, $5-\mu \mathrm{m}$ polystyrene beads (conc. $\sim 3.2 \times 10^{5}$ beads $/ \mathrm{ml}$, from Bangs Lab) are introduced to the microfluidic channel under the same flow condition with PZT actuation operated at $250 \mathrm{~Hz}$ and $9 \mathrm{~V}_{\mathrm{p}-\mathrm{p}}$. For both experiments, videos are taken at the sorting junction and recorded at 3,000 fps. (a)

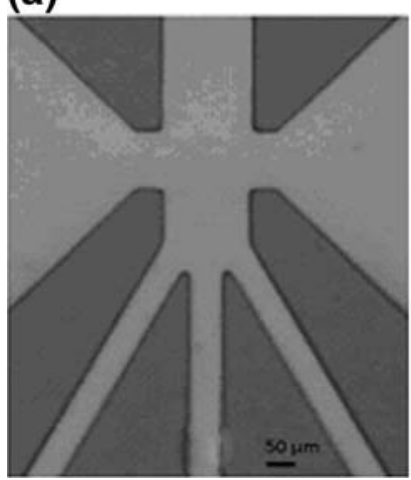

(b)

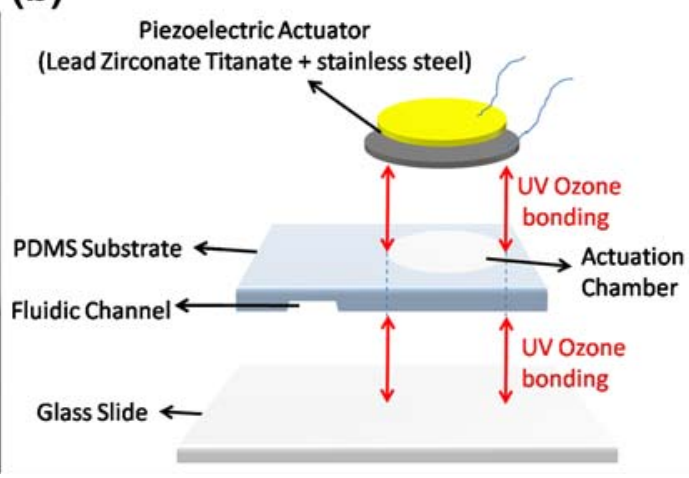

(c)

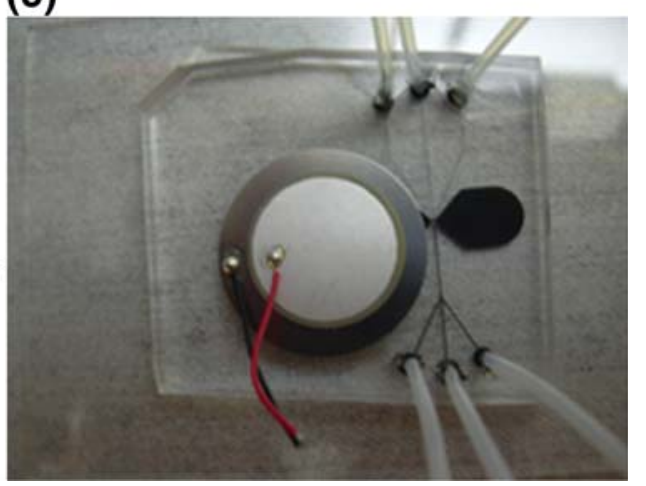

Fig. 2 (a) The device features consist of one $150 \mu \mathrm{m} \times 50 \mu \mathrm{m}$ main channel and three $50 \mu \mathrm{m}$ x $50 \mu \mathrm{m}$ collection channels. The opening of the nozzle (perpendicular to the flow) is $100 \mu \mathrm{m}$ wide. (b) Device fabrication involves two successive bonding using UV-ozone treatment. The resultant device is shown in (c) 


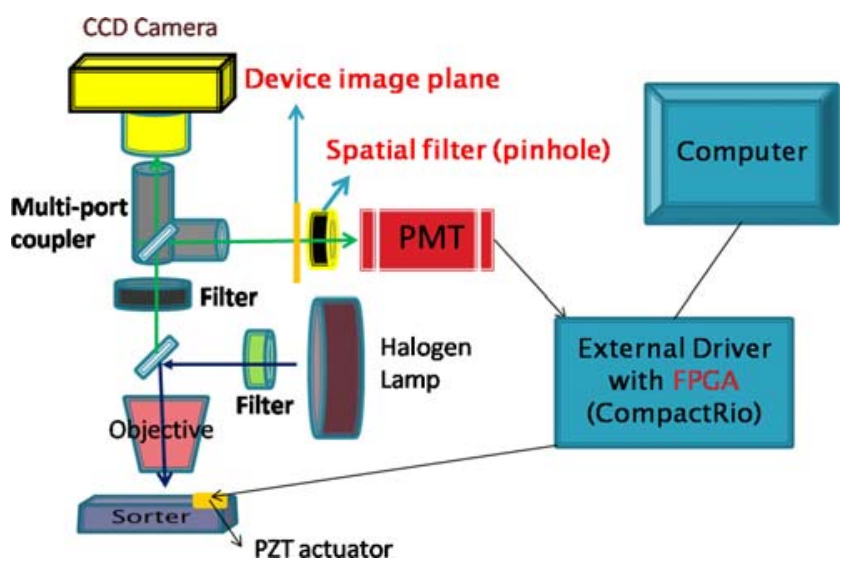

Fig. 3 Schematics of the experimental setup for sorting with closeloop control

\subsection{Deflection of E. Coli cells}

An E. Coli concentration of $\sim 7 \times 10^{5}$ cells $/ \mathrm{ml}$ is prepared and suspended in $1 \mathrm{X}$ PBS solution. For low speed sorting, the total volumetric flow rate is maintained at $2 \mu \mathrm{l} / \mathrm{min}$, and the PZT actuator is operated under $3 \mathrm{Vp}-\mathrm{p}$ at $20 \mathrm{~Hz}$ frequency. To increase throughput, the flowrate is increased to $18 \mu \mathrm{l} / \mathrm{min}$, and the applied voltage and frequency are increased to $6 \mathrm{Vp}-\mathrm{p}$ and $200 \mathrm{~Hz}$. Again, videos are recorded at 6,000 fps, and the sorted E. Coli are enumerated using frame-by-frame images to characterize the cell deflection capability of the device.

\subsection{Automated sorting}

To perform fluorescence-activated cell sorting, a closedloop control system is built (Fig. 3). A $60 \mathrm{~W}$ Halogen lamp is employed as the excitation source. To detect $10-\mu \mathrm{m}$ fluorescent beads (Bangs Laboratory), bandpass filters of 480 and $520 \mathrm{~nm}$ central wavelength are used for filtering excitation and emission lights. After passing through a specially designed spatial filter that allows fluorescence from specific locations in the channel to reach the photomultiplier tube (PMT) detector, the fluorescent emission signal from the cell/particle being sorted is amplified, noisesuppressed, and used to produce a time-delayed actuating signal to drive the actuator, triggering the sorting action. In the following we describe the design and function of the three components critical to close-loop, automated sorting: spatial filter, signal processing (matched filter), and FPGA implementation of signal processing and actuator control algorithms.

A spatial filter allows only fluorescence from certain areas in the channel to reach the detector, thus cutting down the background and crosstalk. Using specially designed patterns, one can spatially encode a fluorescent signal and then transform the signal into a temporarily encoded signal as the targeted particle/cell travels at a speed. We use photolithographic transparency masks (Cad/art services, Inc.) to create spatial filters. The spatially encoded patterns have triple slits and double slits. The former encodes the detection signal and the latter the verification signal from the particles/cells sorted into the designated channel. The width of the slits is $0.25-0.5 \mathrm{~mm}$, translated to $12.5-25 \mu \mathrm{m}$ on the microfluidic channels before magnified by a $20 \mathrm{x}$ microscope objective (Fig. 4).

Real-time electronic control is programmed using Labview (National Instrument) with a programmable external driver (CompactRio, NI). The external driver has an independent operating system with an embedded fieldprogrammable gate array (FPGA) chip. The measured jitter of the system is $<10 \mu \mathrm{sec}$. The flow of the electronic control is shown in Fig. 5. The random high pulse noises of PMT (e.g. caused by sporadic discharge of the device) are removed before running the signal amplification algorithm based on finite impulse response (FIR) filtering. With an FIR matched filter, the signal-to-noise (SNR) ratio can be increased by $18 \mathrm{~dB}$. After SNR enhancement, threshold and search of maximum signal criteria are applied to determine the presence of the detected particle. A signal above threshold indicates that a particle/cell to be sorted is found, triggering the following actions: (a) a delay counter delays the firing of the pulse generator, (b) a preprogrammed output voltage signal is fired to drive the PZT actuator, (c) at certain time period the system is ready to detect the "verification" signal from the sorted sample travelling through the "verification

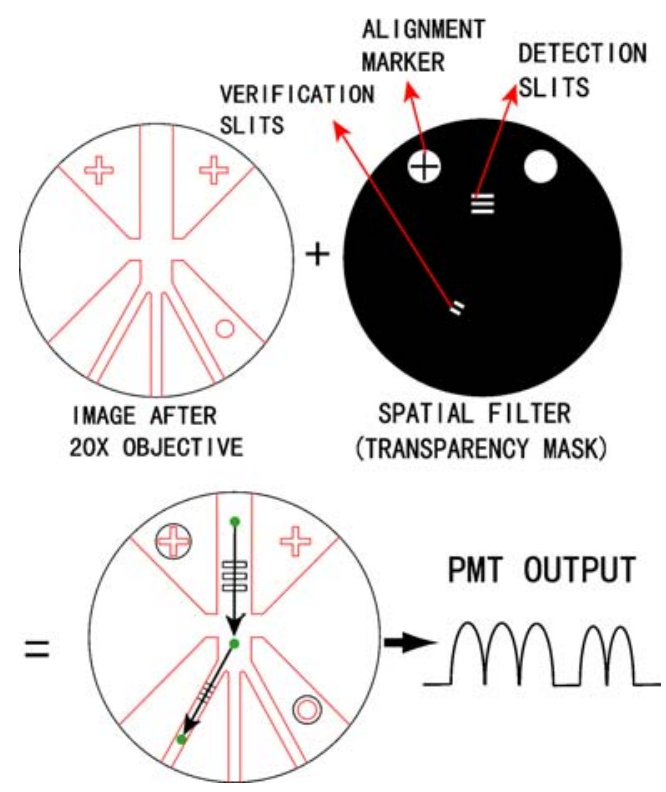

Fig. 4 Design of spatial filter. Spatial filter is designed to purposefully coincide with the image plane after magnification. As fluorescent particle passes through detection slits and gets sorted down to the verification slits, the PMT detector is expected to register signals of 3 peaks followed by 2 peaks 


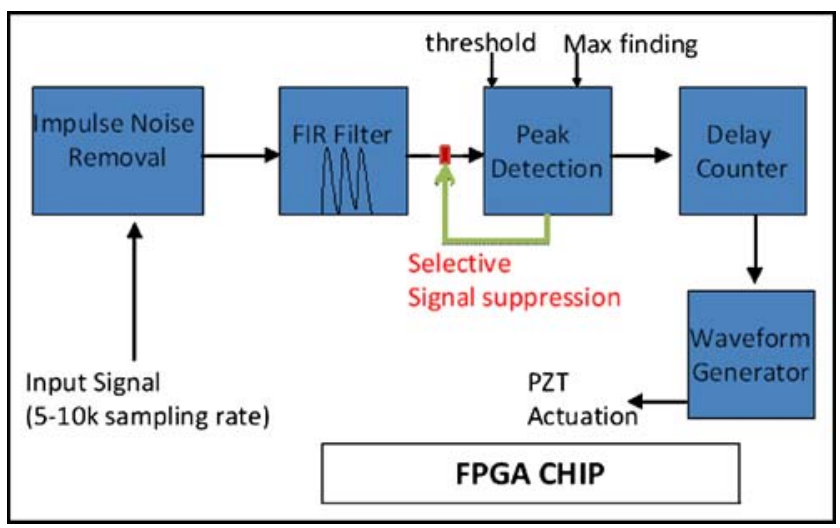

Fig. 5 Flow chart showing the process flow of the electronics control algorithm. The algorithm is programmed into the FPGA chip embedded in the external driver

zone", and (d) update record of the sorting efficiency and sorting error. The amount of time delay equals the travel time of the particle from the optical detection zone to the sorting junction. Until the sorted particle is verified, the PZT actuator will not be fired again. This avoids the problem of confusing the verification signal with the signal of particles traveling too close to the particle being sorted.

\section{Results and discussion}

\subsection{Flow switching capability}

Flow switching of the focused rhodamine stream can be observed under PZT actuation. As PZT actuator pushes the fluid to the left under a rising bias voltage, the rhodamine stream temporarily gets deflected towards the left channel (Fig. 6(a)). Similarly the stream is temporarily shifted towards the right channel as a falling voltage is applied to the PZT actuator (Fig. 6(c)). Without actuation, the stream travels directly down to the center channel (Fig. 6(b)). Such controlled flow switching behaviors are observed at high actuation frequency up to $\sim 1.7 \mathrm{kHz}$ (at $20 \mathrm{~V}_{\mathrm{p}^{-} \mathrm{p}}$ ). The upper limit of the switching frequency has not been experimentally determined yet. However, the channel geometry has been optimized to minimize the viscous effect when subjecting to fluid movement. In order to find out the maximum sort rate at single particle level, further theoretical analysis needs to be investigated to determine the limit of flow response under actuation. Nonetheless, the current study shows that the design can potentially sort several thousand particles/cells per second at single particle/cell resolution.

\subsection{Bead trajectory}

The dynamic simulation shows the trajectory of a $5-\mu \mathrm{m}$ bead subjecting to a time-dependent sinusoidal pressure at the right boundary wall (Fig. 7(b), (d), and (f)). As the particle reaches the sorting junction at a velocity of $5 \mathrm{~cm} / \mathrm{s}$, the pressure force deflects the flow pattern, which in turn carries the particle down to the left collection channel. Particles released at other times (not shown here) can follow different trajectories (e.g. deflected to the right channel or to the center channel) since they experience different pressure force depending on the time they enter the sorting junction. To verify the bead trajectory experimentally, $5-\mu \mathrm{m}$ polystyrene

(a)

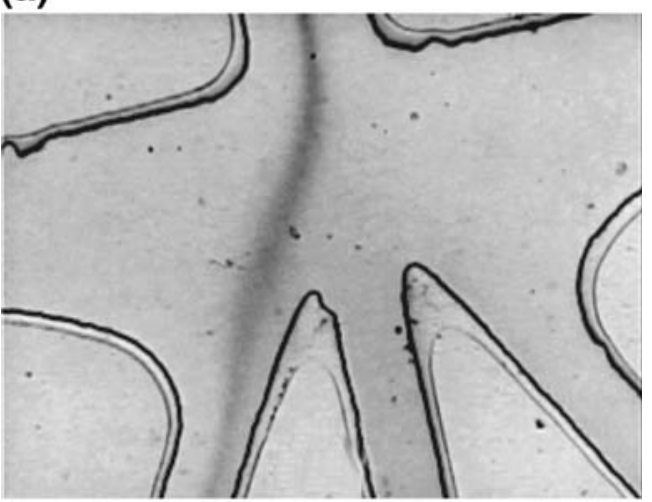

(b)

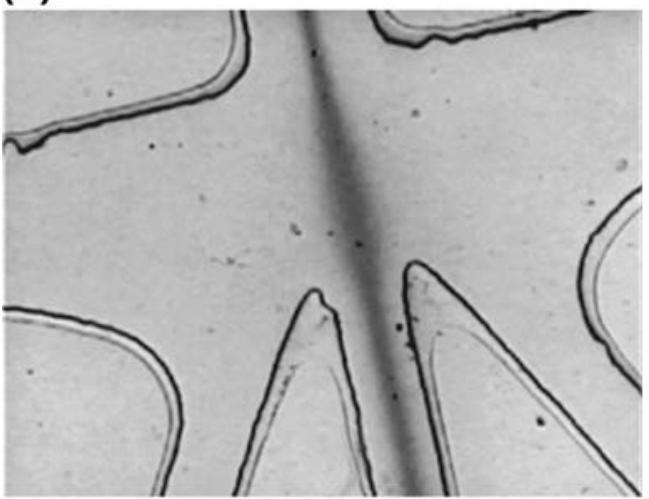

(c)

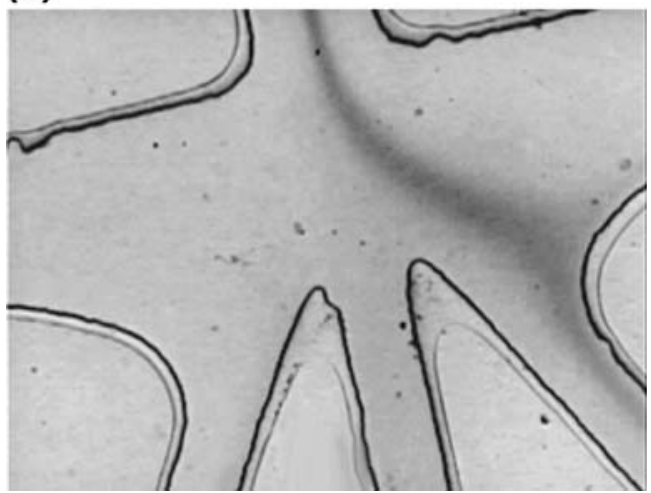

Fig. 6 Images showing deflection of rhodamine dye as a result of PZT actuation. (a) The rhodamine stream switches to the left as the PZT disk bends downward. (b) no stream deflection when the PZT actuator is off. (c) The rhodamine stream is deflected to the right channel as the PZT disk bends upward 
(a)

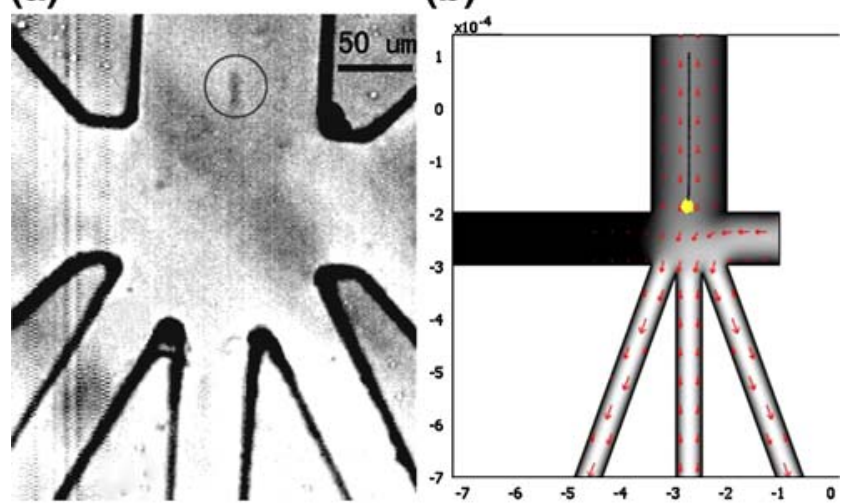

(c)

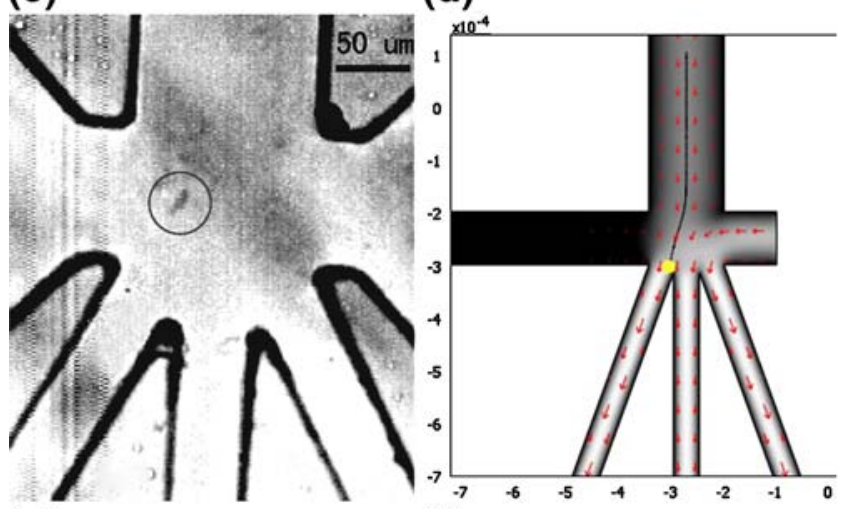

(e)

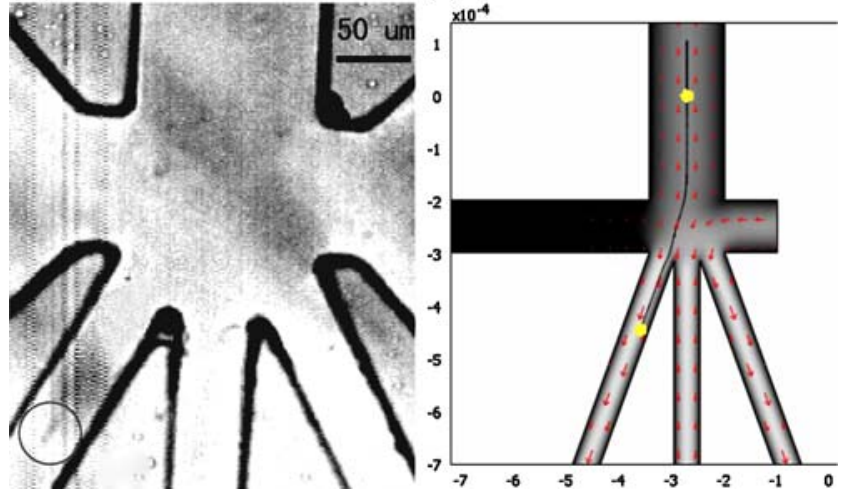

Fig. 7 Sequential images showing particle trajectory based on (b), (d), and (f) dynamic simulation results (time stepping of $1.5 \mathrm{~ms}$ ) and (a), (c), and (e) experimental results (images taken at 0, 1.3, and $3.3 \mathrm{~ms}$ ). The simulation is done by applying $250 \mathrm{~Hz}$ sinusoidal dynamic pressure $(\sim 1.5 \mathrm{kPa})$ to the sorting junction, and the experiment is carried out under $250 \mathrm{~Hz}$ (e.g. sinusoidal) and $9 \mathrm{~V}$ peak-to-peak PZT actuation. Sorted bead is marked for clarity

beads are used. Figure 7(a), (c), and (e)) show how the single bead moves $(\sim 5 \mathrm{~cm} / \mathrm{s})$ when subjected to PZT actuatorinduced fluid disturbance. Similar to the simulation result, as the PZT actuator bends down, the flow imposes a drag force on the bead to deflect the bead off the center stream to the left channel. Also, as the PZT actuator is turned off, the particle immediately resumes its undisturbed trajectory (down the center channel). The result shows that the sorter can impose sufficient drag force to alter particle trajectory in a predictable manner. Furthermore, it shows the promise that the sorter can sort single particles at relatively high speed under low power consumption $(<0.1 \mathrm{~mW})$.

\subsection{E. Coli deflection experiment}

To further characterize the sorting capability of the device, $E$. Coli cells are being introduced to the flow stream under PZT actuation. At $20 \mathrm{~Hz}, 3 \mathrm{~V}_{\mathrm{p}-\mathrm{p}}$ actuation, we analyze the E. Coli. cells that are being deflected into the left and right channels. At a low flow rate of $\sim \mu \mathrm{l} / \mathrm{min}$, a sorting throughput of $24 \mathrm{cells} / \mathrm{s}$ is recorded. To increase the sorting throughput, we increase the total flow rate to $18 \mu \mathrm{l} / \mathrm{min}$, corresponding to a travel speed of $\sim 6 \mathrm{~cm} / \mathrm{s}$ for cells in the center stream, as well as the actuation voltage and frequency to $5 \mathrm{Vp}-\mathrm{p}$ and $200 \mathrm{~Hz}$. At this flow rate, the $E$. Coli cells pass through the sorting junction $(\sim 100 \mu \mathrm{m}$ in length) in $1.7 \mathrm{~ms}$. Figure 8 shows the E. Coli cell deflection result in the aforementioned experimental conditions. Individual peaks represent single cells entering the right/ left collection channels at the specified times. With frameby-frame analysis, we determine the cell deflecting rate to be 330 cells/s. Notice that all the red peaks occur within the rising cycle of the applied voltage, representing samples entering the left channel. Similarly, all the blue peaks occur

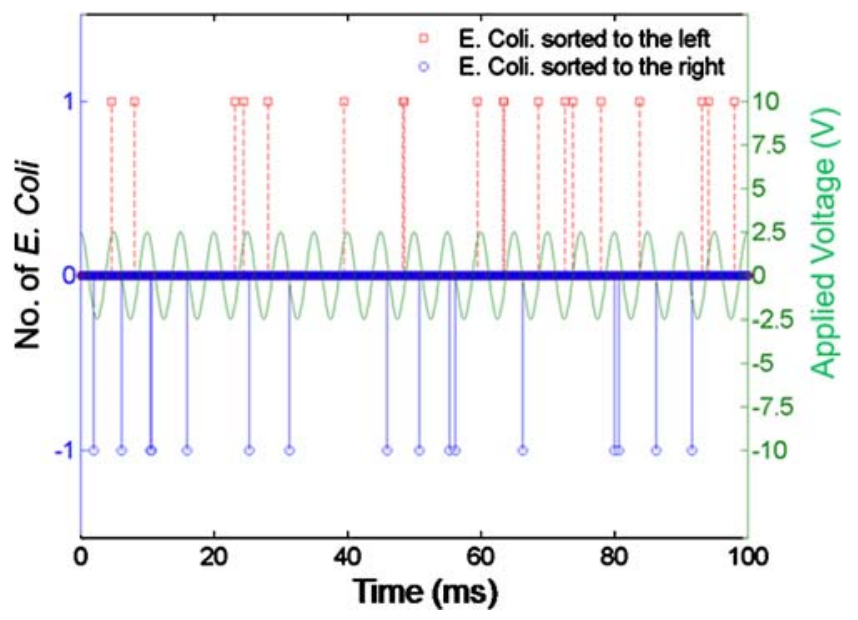

Fig. 8 Deflection of single E. Coli. cells at $200 \mathrm{~Hz}$ frequency and 5 Vp-p actuation voltage. The peaks are obtained by identifying cells visually as they are sorted to the left/right channels. Approximately 18 and $17 \mathrm{E}$. Coli. cells are sorted to the left/right in $100 \mathrm{~ms}$ in this case. However, a total of 330 cells are visually counted in $1 \mathrm{sec}$. Note that the cells that are sorted to the left/right all fall into downward/upward (bending down/up) ramping state of the PZT actuator, in good agreement with the theory. Also note that few rare peaks that appear denser mean that two cells can exit a particular collection channel at roughly the same time 


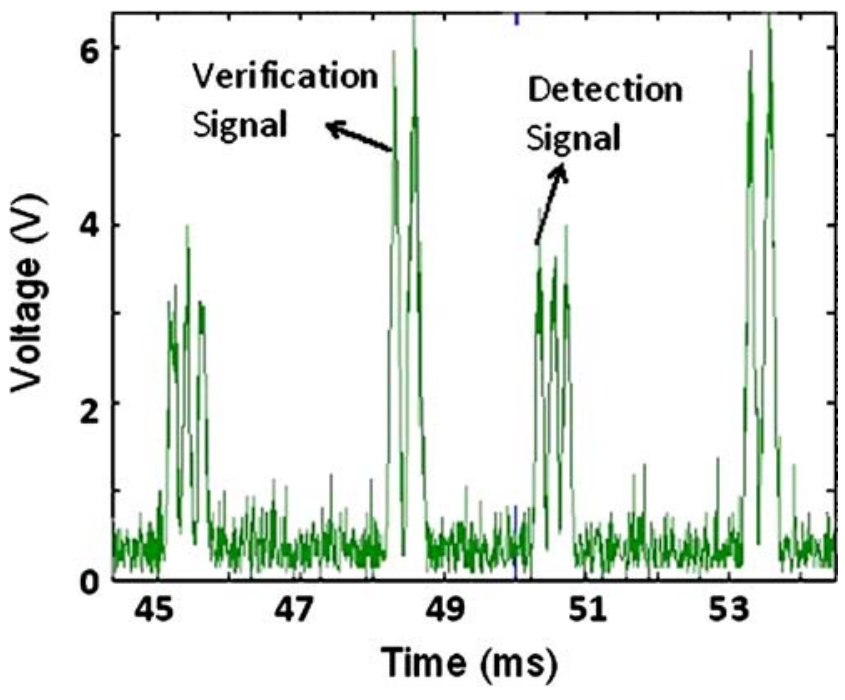

Fig. 9 Raw signals of $10-\mu \mathrm{m}$ beads passing through the 3-slit upstream detection zone and 2-slit downstream verification zone. Every peak results from particle passing through individual slits (slit width $=17.5 \mu \mathrm{m}$ ). Note that for the two particles shown here, the time for them to travel from the detection region down to the verification region is $\sim 2 \mathrm{~ms}$

within the falling cycles of the applied voltage, representing samples entering the right channel. This is in total agreement with the proposed sorting mechanism that a rising (falling) voltage bends the PZT downward (upward) to create a transient transverse flow towards the left (right) channel, which in turn directs the cells into the desired collection channel.

\subsection{Noise suppression by spatial filter and signal processing}

As a $10-\mu \mathrm{m}$ fluorescent bead crosses the detection slits and is sorted to pass the verification slits, a 3-peak signal followed by a 2-peak signal are observed (Fig. 9). The distinctive nature of the peaks allows real-time monitoring of sorted and unsorted particles, which, to our best knowledge, has never been implemented in any $\mu$ FACSs. The detected signals can be amplified using a real-time signal processing algorithm based on the concept of match filter. The mathematical model of the FIR match filter can be described as

$Y[n]=\sum_{k=0}^{M} b_{k} \cdot x[n-k]$

where $Y[n]$ is the output signal, $x[n]$ is the input signal, $b_{k}$ is the filter coefficients, and $M$ is the filter order, which specifies the number of taps (e.g. $M+1$ ) for the filter coefficients. Thus, in the proposed design, the match filter coefficients, which assume the shape similar to the detected signals (e.g. 3 sinusoidal lobes), are used to convolve with the detected signal, resulting in SNR enhancement of $\sim 18 \mathrm{~dB}$, as shown in Fig. 10.

\subsection{Sorting efficiency and sorting accuracy}

To characterize the sorting efficiency, $10-\mu \mathrm{m}$ fluorescent beads (Bangs Laboratory) with a concentration of $\sim 1 \times 10^{5}$ particles $/ \mathrm{ml}$ and excitation/emission wavelength of 480/ $520 \mathrm{~nm}$ are used. The beads are hydrodynamically focused under a sample-to-sheath flow ratio of 1:2 with velocities of $\sim 8 \mathrm{~cm} / \mathrm{s}$. Square pulses of $10 \mathrm{~V}_{\mathrm{p}-\mathrm{p}}(1.5 \mathrm{~ms}$ pulse width $)$ are used to drive the PZT actuator. The delay time $(\sim 1.5 \mathrm{~ms})$ is estimated according to the velocity of the particle. The measurement data are recorded for a total period of $10 \mathrm{~s}$. We use the setup to measure the sorting efficiency and the sorting error. The former refers to the success rate of a voltage pulse to direct the targeted particle into the designated channel; and the latter refers to the possibility for an unintended particle to enter the designated channel by mistake. A sorting efficiency less than $100 \%$ could be due to non-ideal timing control, limited flow response, or timing jitter. In most practical applications, this is less a concern than sorting error. On the other hand, a sorting error, caused likely by non-ideal flow disturbance due to actuation, could have a much more adverse effect. Therefore, a strategy is taken to achieve the lowest possible sorting error at some expense of sorting efficiency. From a subset of the measurements, Fig. 11 shows a sorting efficiency of $\sim 70 \%$. Other measurements consistently show similar results $(70 \% \pm 5 \%)$. The distinction between successfully and unsuccessfully sorted particles is by examining the presence and absence of the verification signal following the first detected signal. The verification signal is designed to have a lower intensity than the first detected signal because the spatial filter for the verification signal has only two slits, producing a less match to the triple-peak FIR filter. Nonetheless, the amount of SNR enhancement

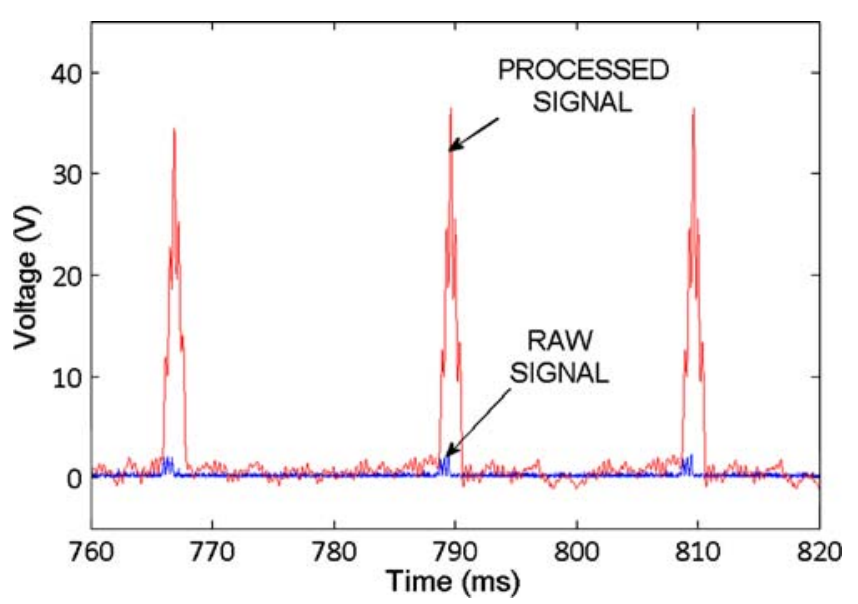

Fig. 10 A comparison between raw and amplified signals with FIR match filter. The amplified signal shows a sensitivity enhancement of $\sim 18 \mathrm{~dB}$ 
Fig. 11 Sorting efficiency and sorting error characterization. 44 beads (blue dots) out of 64 detected fluorescent beads have been successfully sorted, resulting in $\sim 70 \%$ sorting efficiency. For every single sorted particle, the detected signal (upstream) is always followed by a verification signal (downstream). In contrast, the absence of verification signals results in particles not being sorted. In this experiment, there is $0 \%$ sorting error since no particles have been falsely sorted

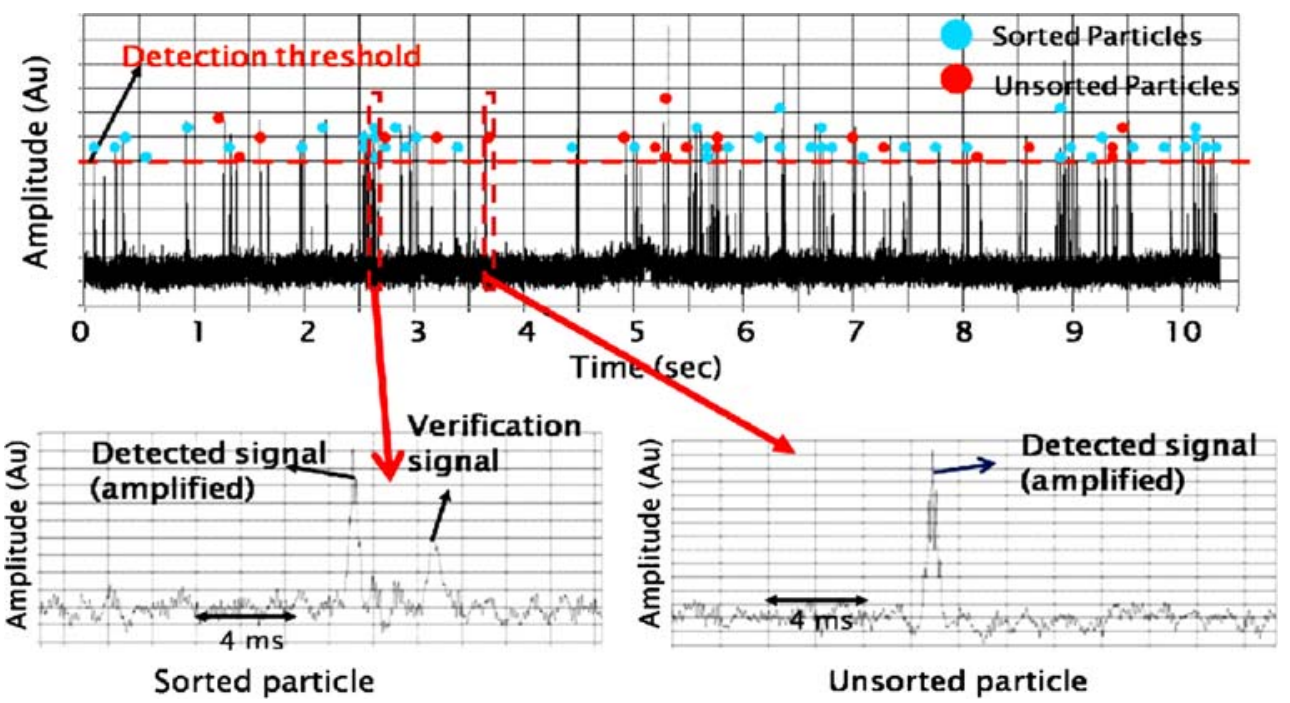

for the verification signal still allows clear identification. In the instance that two particles are spaced closely such that the second particle enters the detection region before the first particle is being verified downstream, to avoid misfiring of PZT actuation due to cross-talking between detection and verification signals, our algorithm is set up to keep the actuator inactive until the first particle is sorted and verified. This holding period ( $\sim 3-4 \mathrm{~ms})$ also contributes to the reduced sorting efficiency. From the data in Fig. 11 and a series of preliminary measurements, we have achieved $0 \%$ sorting error and the estimated minimum particle spacing to ensure error-free sorting is $\sim 100 \mu \mathrm{m}$.

The current experiment uses filtered mercury lamp as the fluorescence excitation source. To increase sensitivity for sorting of fluorescently-tagged biological agents, $488 \mathrm{~nm}$ laser $(\sim 40 \mathrm{~mW})$ will be used as the excitation source. These developments, in conjunction with integration of optical devices with microfluidic channels, could lead to highthroughput, high efficiency, and high accuracy microfabricated cell sorter at the single cell level.

\section{Conclusion}

We have demonstrated, for the first time, a microfluidic cell sorter with integrated piezoelectric actuator. The device is easy to fabricate and operates at less than $10 \mathrm{Vp}$-p. In the experiment of instantaneous flow switching, we have shown that the flow stream responds to the piezoelectric actuator at high frequency $(\sim 1.7 \mathrm{kHz})$ and the amount of deflection of cells/particles in the flow can be precisely controlled. Both simulation and experiment show that particles of any size, shape, and density of interest can be individually sorted in a controlled manner. In the experiment of $E$. Coli deflection, a sinusoidal voltage deflects cells at a rate of 330 cells/s and shows a highly repeatable operation consistent with the theory. Using a specially design spatial filter and a real-time signal processing algorithm implemented in FPGA, a closed-loop sorting system is demonstrated with zero error rate and a sorting efficiency of around $70 \%$. Compared with other $\mu \mathrm{FACS}$, our sorting system has advantages in three areas. First, the spatial filter design and the real-time signal processing algorithm enhance the signal-to-noise ratio by $18 \mathrm{~dB}$ and allow verification of sorting. Second, the PZT-actuated sorting module is easy to fabricate, consumes minimum power $(0.1 \mathrm{~mW})$, operates at low voltage $\left(<10 \mathrm{~V}_{\mathrm{pp}}\right)$, and has a much faster response $(0.1-1 \mathrm{~ms})$ than off-chip mechanical actuators such as check-valves and syringe pumps. Third, the FPGA-based electronics control enables real-time signal amplification, user-defined delay time, programmable output waveform, and low timing jitter $(<10 \mu \mathrm{sec})$. These features contribute significantly to a low-cost sorter that can perform high-throughput cell sorting at a single-cell level.

Acknowledgement The research is supported by the NIH grants 1R21RR024453-01 and 1R01HG004876. We would like to thank the Nano3 staff for their technical support.

Open Access This article is distributed under the terms of the Creative Commons Attribution Noncommercial License which permits any noncommercial use, distribution, and reproduction in any medium, provided the original author(s) and source are credited. 


\section{References}

H. Andersson and A.v.d. Berg, Sensors and Actuators B 92, 315 (2003)

H. Bang, C. Chung, J.K. Kim, S.H. Kim, S. Chung, J. Park, W.G. Lee, H. Yun, J. Lee, K. Cho, D.-C. Han, J.K. Chang, Microsyst Technol 12, 746 (2006)

G. Blankenstein, U.D. Lasen, Biosens Bioelectron 13, 427 (1998)

T. Braschler, N. Demierre, E. Nascimento, T. Silva, A.G. Oliva, P. Renaud, Lab Chip 8, 280 (2007)

J.M. Coulson, J.F. Richardson, J.R. Backhurst, J.H. Harker, Chemical engineering, 2nd edn. (Butterworth-Heinemann Ltd, Oxford, 1982), p. 970

P.S. Dittrich, Anal Chem 75, 5767 (2003)

I. Doh, Y.-H. Cho, Sens. and Actuators A 121, 59 (2005)

S. Fiedler, S.G. Shirley, T. Schnelle, G. Fuhr, Anal Chem 70, 1909 (1998)

A.Y. Fu, C. Spence, A. Scherer, F.H. Arnold, S.R. Quake, Nat Biotech 17, 1109 (1999)

A. Y. Fu, California Institute of Technology, Pasadena (thesis). 115 (2002)
A.Y. Fu, H.-P. Chou, C. Spence, F.H. Arnold, S.R. Quake, Anal Chem 74, 2451 (2002)

L.-M. Fu, R.-J. Yang, C.-H. Lin, Y.-J. Pan, G.-B. Lee, Analytica Chimica Acta 507, 163 (2004)

D. Holmes, M. E. Sandison, N. G. Green, H. Morgan, Nanobiotechnol. (2005) doi:10.1049/ip-nbt:20050008

X. Hu, P.H. Bessette, J. Qian, C.D. Meinhart, P.S. Daugherty, H.T. Soh, PNAS 102, 15757 (2005)

J. Krüger, K. Singh, A. O’Neill, C. Jackson, A. Morrison, P. O’Brien, J Micromech Microeng 12, 486 (2002)

B.H. Lapizco-Encinas, B.A. Simmons, E.B. Cummings, Y. Fintschenko, Electrophoresis 25, 1695 (2004)

G. D. Meredith, C. E. Sims, J. S. Soughayer, N. L. Allbritton, Nat Biotech 18, 309 (2000)

N. Pamme, Lab Chip 6, 24 (2006)

N. Pamme, C. Wilhelm, Lab Chip 6, 974 (2006)

N. Pamme, Lab Chip 7, 1644 (2007)

C.E. Sims, N.L. Allbritton, Lab Chip 7, 423 (2007)

A. Wolff, I.R. Perch-Nielsen, U.D. Larsen, P. Friis, G. Goranovic, C.R. Poulsen, J.P. Kutter, P. Telleman, Lab Chip 3, 22 (2003)

Y. Xia, G.M. Whitesides, Annu Rev Mater Sci 28, 153 (1998) 